\title{
The Grounds and the Components of Concepts
}

\author{
Jan Claas $^{1}$ (D)
}

Received: 31 August 2020 / Accepted: 2 September 2021

(c) The Author(s) 2021

\begin{abstract}
In this paper I investigate the idea that in conceptual analysis we are in a substantial way concerned with revealing metaphysical grounds. I argue that a recent proposal fails, according to which we aim to reveal what complex concepts are grounded in. The notion of composition, rather than that of grounding, is the best way to understand the intuitive hierarchy of concepts. In an analysis we reveal the components or parts of complex concepts and their structure. Finally, I propose an alternative role for grounding in our accounts of analysis: in analyses we reveal truths about the composition of concepts that serve as grounds for truths about their functions, which are what we want to understand.
\end{abstract}

\section{Introduction}

According to an idea which was especially popular at the beginning of the 20th century, much of philosophy is rigorous engagement with concepts: philosophers investigate our main representational devices by analyzing them (Glock, 2008, ch. 2). Nowadays, it is often assumed that conceptual analysis has a rather poor track record and that it faces important challenges (e. g., Fodor, 1998; Kornblith, 2002, ch. 1; Williamson, 2007). Nevertheless, it still is considered to be a philosophical core investigation. Most notably, it currently plays an important role in the debate on conceptual engineering (cf. Eklund, 2015; Ball, 2020; Pettit, 2020).

According to another idea which is especially popular now, at the beginning of the 21st century, much of philosophy is rigorous engagement with metaphysical grounds (cf. Correia and Schnieder, 2012; Raven, 2020): philosophers investigate non-causal grounds of phenomena, they find out in virtue of what things are as they are and they try to capture the structure of reality. Adherents of this approach also face important challenges (e. g., Daly, 2012; Wilson, 2014; Maurin, 2019; Koslicki, 2020), including concerns about the intelligibility and usefulness of the notion. Nevertheless, the progress philosophers are making in understanding grounding is

Jan Claas

jan.claas@univie.ac.at

1 Department of Philosophy, University of Vienna, Universitätsstraße 7, 1010 Vienna, Austria 
noteworthy and grounding has far-reaching applications. It allows us to reframe traditional debates and problems such as the mind-body problem (Bryant, 2020) and to revisit traditional notions such as essence (e. g., Fine, 2015; Zylstra, 2019).

The attention these two kinds of investigations have received raises the question of whether they are substantially related. A connection would indeed be very welcome as it would help to improve our understanding of both notions, which still are in need of clarification. Fortunately, we have reasons to suspect that grounding and analysis are not alien to one another. Both notions are tightly linked to the notion of explanation and we reveal hierarchies, how certain things are ordered according to an intuitive notion of fundamentality. Given these resemblances, it is hardly surprising that interrelations already have been discussed. ${ }^{1}$ A strong way for such a connection to obtain would be if one of the two is to be understood in terms of the other. The aim of this paper is to discuss specific ways to understand the following claim, which suggests one such connection:

Grounding Conceptual Analysis Conceptual analyses are investigations into metaphysical grounds.

Thereby we would understand conceptual analysis in terms of grounding. ${ }^{2}$ Different ways of fleshing this idea out can be seen as explications of different ways to understand analysis in terms of grounding.

I begin with an introduction of grounding and conceptual analysis in Sect. 2. I then discuss two ways to understand analysis in terms of grounding that are concerned with revealing grounds of complex concepts. According to the recently proposed concept grounding view, an important part of analyses is to reveal the grounds of complex concepts; in Sect. 3 I argue that the concept grounding view fails. Grounding relations, however, arise from a traditional understanding of complex concepts as composite objects, which is what I will argue for in Sect. 4. In Sect. 5 I present a limited task for grounding in analysis: in analyses we aim to understand certain functions of concepts, which they have in virtue of their composition.

\footnotetext{
${ }^{1}$ We can find explicit connections, e. g., in Leibniz $(1989,337)$ and Bolzano (1849). For contemporary discussions see Schnieder (2010) and Horvath (2018), which I will discuss in detail in Sect. 3.

${ }^{2}$ If we want to understand grounding in terms of conceptual analysis, we have to consider:

Conceptually Analyzing Grounds Investigations into metaphysical grounds are investigations into the configuration of concepts. While I have to put this claim aside, it also deserves our attention and it has a noteworthy proponent (Leibniz 1989, 337).
} 


\section{Grounding and Analysis}

\subsection{Grounding}

Grounding is often assumed to be an objective explanatory relation of metaphysical dependence between entities such as truths or facts. It is usually expressed by certain uses of "because" and "in virtue of". With "Daisy is an animal because Daisy is a duck" I express that the truth (or fact) that Daisy is a duck is a ground of the truth (or fact) that Daisy is an animal. I also might have expressed it by stating that Daisy is an animal in virtue of being a duck. Taking truths, understood as true propositions, to be the relata of grounding is one common option. Another one is to consider grounding to be a relation between facts, which may be understood as truths' more coarse-grained worldly counterparts (Roski and Schnieder 2019, 682f). For this paper the choice between the two options is purely terminological and I will usually prefer truth-terminology. A third option, entity-grounding, will be discussed for concepts in particular in Sect. 3.2.

A relatively uncontroversial case of grounding is grounding true conjunctions in their conjuncts. A true grounding claim is expressed, for example, by "Socrates is an Athenian and a philosopher because Socrates is an Athenian and Socrates is a philosopher", stating that the truth that Socrates is an Athenian and a philosopher is grounded in the truth that Socrates is an Athenian and in the truth that Socrates is a philosopher (cf. Bolzano, 1837, vol. II, §199, p. 344; Fine, 2012, 58). Also, instances of Aristotle's Insight such as "It is true that you are pale because you are pale" (cf. Aristotle, 1984, Metaphysics IX, §10, 1051a34-1051b17) are commonly taken to express grounding relations (Correia and Schnieder, 2012, 26). Philosophers in the debate also claim that

1. truths stating instantiations of determinables are grounded in truths stating instantiations of their respective determinates (e. g., Rosen, 2010, 126-130; Audi, 2012b; Trogdon, 2018, 1291), that, e. g., "This blossom is blue because it is azure" is a true grounding statement;

2. truths stating dispositional properties are grounded in truths stating categorical properties (e. g., Rosen, 2010, 110; Audi, 2012b), that, e. g., glass is brittle in virtue of having a certain particle-structure;

3. the existence of wholes is grounded in the existence of their parts (e. g., Cameron, 2014; Loss, 2016), that, e. g., the truth that my bicycle exists is grounded in the truth that its frame exists.

These examples and the characterization as objective explanatory relation between truths gives us, via our understanding of an important usage of "because" and the notion of explanation, an idea of what grounding is supposed to accomplish. As I 
take this to be the best way to understand and motivate grounding, I rely on tight links between grounding, explanations and why-questions. ${ }^{3}$

Let me turn to the formal features of grounding according to what I take to be the standard view. As a relation between truths or facts, grounding is a factive relation. Furthermore, grounding is irreflexive and asymmetric: no truth is its own ground and what is grounded in a truth cannot also be its ground. Most grounds have grounds themselves, the other few are fundamental, i. e. ungrounded, truths. So we often have grounding chains and can thereby distinguish between immediate grounding, which is not transitive, and mediate grounding, which is the transitive closure of immediate grounding. Importantly, we can also distinguish between full and partial grounds: for many truths we need several truths to give their full ground. The individual truths that make up a full ground of a given truth are its partial grounds. A conjunction, for example, is partially grounded in each of its conjuncts. ${ }^{4}$

\subsection{Analysis}

Compared to the debate on grounding there is less agreement on the central features of conceptual analysis. Fortunately, all we need to presuppose for the following is a minimal and broad understanding. It should be compatible with most views that share the general idea that in analyses we "unpack" or "break down" the analysandum in some sense that is further to be explicated. The basic idea is naturally spelled out in traditional terms of complexity and containment: there are complex concepts and simpler concepts such that some of the latter in some way figure in or are "contained" in the former. These are intuitive metaphors and an account of analysis needs to clarify what they hint at by providing necessary and sufficient conditions.

Importantly, analyses reveal simpler concepts that in some way figure in a given complex one and the way in which they do so. Analyses yield a better understanding of complex concepts insofar as simpler concepts and their features often account for certain features of the complex concept. One such feature is to specify application conditions, which are the properties objects must have in order to fall under the complex concept, i. e. for the concept to "represent" these objects. For instance, in an analysis the concept DRAKE should turn out to be the conjunction of MALE and DUCK with the latter two concepts specifying application conditions of DRAKE; JADE is a candidate for a disjunctive concept, which is analyzed into NEPHRITE and JADEITE.

According to such an understanding, we have an analysis of a concept $\mathrm{C}$ only if

\footnotetext{
${ }^{3}$ Links of this kind are commonly but not universally assumed in the literature. For ways of spelling these links out, see, for example, Schnieder (2010), Audi (2012a), Skow (2016); see Wilson (2014) and Maurin (2019) for important worries.

${ }^{4}$ See Correia and Schnieder (2012), Fine (2012), Bliss and Trogdon (2016) for statements or summaries of the standard view. Concerning most features, the majority of contemporary accounts agrees with Bolzano (1837, vol. II, §162, 191-194, §198-222, 339-391). For contemporary views deviating from or modifying the standard view, see, e. g., Jenkins (2011) for irreflexivity, Thompson (2016) for asymmetry and Schaffer (2012) for transitivity.
} 
1. the concepts into which $\mathrm{C}$ is analyzed are less complex than $\mathrm{C}$;

2. the combination of the concepts into which $\mathrm{C}$ is analyzed has, in the configuration indicated by the analysis, necessarily the same extension as $\mathrm{C}$.

But these two necessary conditions are not jointly sufficient. While EQUIANGULAR TRIANGLE and EQUILATERAL TRIANGLE necessarily represent the same objects, an analysis of the former should reveal EQUIANGULAR rather than EQUILATERAL, which instead should appear in an analysis of EQUILATERAL TRIANGLE. So not all simpler concepts that specify application conditions for a concept figure in its analysis. In order to satisfactorily account for analysis, one important task is to provide a further condition, which presumably captures a further relation between the analysans and the analysandum.

\section{Grounding Concepts}

Filling this gap in our accounts of analysis is Horvath's (2018) motivation for proposing a specific way to understand analysis in terms of grounding, the concept grounding view, which I will discuss in detail for two reasons. For one thing, it is a clear statement of the idea that in analyses we reveal what complex concepts are grounded in, which is a salient way to understand analysis in terms of grounding. And it serves as a case study. The concept grounding view applies a specific strategy to argue for grounding relations. According to this strategy, when we need to account for a phenomenon and we cannot do so with the notions at hand, especially if modal notions are not fine-grained enough, then we may assume that the things involved stand in grounding relations to each other if this allows us to account for the phenomenon. Wilson (2014) has argued that this strategy generally fails as grounding does not really perform the tasks it is invoked for. While I am less pessimistic about the tenability of the strategy, in this section I indeed argue that it fails in this particular case.

A terminological note: I prefer to talk about conceptual analysis, Horvath prefers to talk about philosophical analysis. Whatever important differences there are between our understandings of analysis, they are not important for my discussion of his proposal. We agree that the intuitive priority relation that obtains, e. g., between the concept FOAL and the concept HORSE, is the key to a better understanding of analysis while we disagree on which relation it should be identified with.

\subsection{The Concept Grounding View}

The concept grounding view assumes entity-grounding: all kinds of objects, not just truths or facts, can ground each other. In order to understand the proposal, let us assume that other things than truths or facts can stand in such explanatory relations to each other until I discuss the choice of grounding-relata in Sect. 3.2. According to the proposal, complex concepts are mediately grounded in the concepts that are revealed in an analysis and immediately grounded in tuples of them. Tuples are 
required in order to account for the structure at least some complex concepts seem to exhibit (Horvath 2018, 734-736). The concept BACHELOR, for example, is immediately grounded in the ordered pair < UNMARRIED, MAN $>$ and mediately in UNMARRIED and MAN. It also can be immediately grounded in the ordered pair <MAN, UNMARRIED>, as one grounded can have multiple distinct full grounds. Although tuples do not usually play an explicit role in our practice of analyzing concepts, they are an essential part of the proposal. Consider concepts that are ultimately analyzed into the same concepts such as UNFORTUNATE AND COMMON EVENT and FORTUNATE AND UNCOMMON EVENT. We do not want these concepts to have exactly the same analysis as they do not even have the same extension, even though the concepts that figure in them are exactly the same, at least at a sufficiently fine-grained level of analysis: FORTUNATE, COMMON, EVENT and a negation concept, which combines with FORTUNATE in one and with COMMON in the other concept. The difference can be accounted for, in the proposal, by distinguishing the tuples that are the immediate grounds on basis of the position of the negation concept.

According to this view, in analyses we investigate grounds of concepts as we investigate which concepts in which tuples complex concepts are grounded in. While the extension of EQUILATERAL TRIANGLE can be fixed in terms of EQUILATERAL and TRIANGLE as well as of EQUIANGULAR and TRIANGLE, it plausibly is grounded only in the former pair of concepts, which is why EQUILATERAL rather than EQUIANGULAR appears in the analysis. In a similar vein we can apply concept-grounding in order to satisfy a variety of desiderata (Horvath, 2018, 741-743). According to the concept grounding view, we then should believe in concept-grounding because it gets the job done: it fills a vexing gap present in our accounts of analysis.

To be more precise, the motivation to believe in concept-grounding has to be that it is the best candidate that completes the account. Intuitively, some concepts are more fundamental than others and grounding has the correct structural features to account for the intuitive hierarchy of concepts (Horvath, 2018, 732-734). Besides the features mentioned above that make it a strict partial order, grounding filters out irrelevant concepts via an intuitive notion of relevance. However, not every intuitive hierarchy is to be understood in terms of grounding and other relations share the features required for the task at hand. There are several strict partial orders that sort their relata according to intuitive notions of relevance and fundamentality. ${ }^{5}$ They then may account equally well for the intuitive hierarchy while they are more or less attractive when we consider the overall costs involved. So having the features mentioned makes concept-grounding $a$ candidate. If it is not the only candidate, the concept grounding view has to show that it is the best candidate.

The rival candidate I focus on is to treat complex concepts as composite entities and let the notion of composition do the work instead of grounding. According to such an understanding, an analysis reveals the components of a complex concept and the structure in which these components together compose the concept. In its bold

\footnotetext{
5 Further candidates can be found among Bennett's building relations (Bennett, 2017). Building relations are relations that explain how more fundamental things account for less fundamental things, among them grounding and mereological composition but also, e. g., set-formation.
} 
versions, the mereological terminology often used in order to characterize concepts is understood literally: complex concepts are related to their components as wholes are related to their parts. Mereological principles then generally apply to concepts and their components. Relying on a mereological notion of composition is a viable idea for the task at hand. According to our core understanding, proper parthood also is a strict partial order (Varzi, 2016, sect. 2) and it displays a sensitivity to only consider relevant entities as parts in the first place. So we have two promising candidates to complete our gappy account of analysis:

Concept-grounding Simpler concepts are the grounds of complex concepts. Complex concepts are grounded in the concepts that are revealed in an analysis.

Concept-composition Simpler concepts are mereological or non-mereological components of complex concepts. Complex concepts are composed of the concepts that are revealed in an analysis.

The concept grounding view raises objections against concept-composition, which I will discuss in Sect. 4. But even if concept-composition is not refuted by these objections, one still may prefer concept-grounding for another reason: the notion of composition involved has "stronger commitments in the metaphysics of concepts" (Horvath, 2018, 736) than the notion of grounding. ${ }^{6}$ Intuitively, it "takes more" for DUCK to compose DRAKE, especially if this also means that DUCK is a part of DRAKE, than for DUCK to just ground DRAKE in one way or another. If DUCK composes DRAKE, then, it seems, DUCK also grounds DRAKE but if DUCK grounds DRAKE, then, it seems, DUCK does not have to also compose DRAKE. Nevertheless, we should, as I will argue, prefer concept-composition. But first a clarification concerning the relata of grounding is in order.

\subsection{First Worries: Entity-Grounding and the Tuple-Detour}

I introduced grounding as a relation between truths or facts, not between concepts and tuples or concepts and concepts. Talking in the latter way may either be taken as a parsimonious way of expressing oneself or as suggesting that concepts and tuples are also relata of grounding, which is the case in the concept grounding view. However, being permissive in this respect is not unproblematic and raises a first worry.

While it indeed has been proposed that all kinds of things stand in grounding relations (Schaffer, 2009, 2010), there are reasons to restrict the class of grounding relata. One reason why we should prefer a more careful approach is an intelligibility worry. ${ }^{7}$ Truths or facts intelligibly stand in explanatory relations to each other in a way plain objects, prima facie, do not. Facts and truths typically involve that some objects are or act in some way or that they exist; giving a ground then

\footnotetext{
6 This objection can seem puzzling if one believes that we are already committed to treat complex concepts as composite objects, which many philosophers seem to be (see Sect. 4). Relying on concept-composition then would not come with any additional commitments.

7 See Sider (2012, ch. 8.6) and Koslicki (2015) for similar worries.
} 
is to state truths or facts why they do so. It is intuitively clear what the ground for the truth or fact that my breakfast had 400kcal looks like. It is another truth or fact such as the one that it consisted of an avocado with 400kcal. However, it can be difficult to even comprehend what the ground of just my breakfast is supposed to be. Put in terms of explanation: we can explain why the concept HORSE is a difficult concept and maybe why it represents horses. We cannot explain, in the same sense, the concept HORSE.

In order to deal with this worry about concept-grounding, it is not enough to argue for the intelligibility of grounding in general. The fact that I can peel onions indicates that the notion of peeling is intelligible and that the activity peeling exists. It does not indicate that the notion of water-peeling is intelligible or that the activity water-peeling exists, even if onion-peeling, a proper instance of peeling, gives us a hunch of what might be involved in water-peeling. So even if we understand grounding because we understand truth- or fact-grounding, concept-grounding can be unintelligible. The challenge is to give skeptics about entity-grounding a way to understand the concept grounding view in case they understand truth- or fact-grounding. As the intelligibility worry concerns a particular way of construing concept-grounding, we can construe it as a relation between truths or facts about concepts by making explicit what about concepts is supposed to be grounded.

The salient and least invasive strategy is the "existential paraphrase strategy" (Koslicki, 2015, 328). The concept grounding view approves of this strategy in case questions must be answered concerning the "metaphysical 'aspect' " (Horvath, 2018,737 ) of concepts that is relevant for concept-grounding. The existential paraphrase would be: the truth that the concept BACHELOR exists is grounded immediately in the truth that the ordered pair <UNMARRIED, MAN> exists. Mediately, it is grounded partially in the truth that UNMARRIED exists and partially in the truth that MAN exists.

Once we apply the existential paraphrase strategy, it becomes quite apparent that relying on tuples as immediate grounds is not very intuitive. While the truth that BACHELOR exists may intuitively be grounded in truths stating the existence of UNMARRIED and MAN, the truth that the tuple <UNMARRIED, MAN> exists seems ill-suited to help explain the existence of BACHELOR. In order to provide an explanation for the existence of something complex it is natural to rely on the existence of the relevant less complex objects. It is not a natural idea to additionally introduce a third entity in which all of the less complex objects figure but which is not, and by the irreflexivity of grounding cannot be, identical to the complex object. Not only is there no independent reason to assume so, it is an implausible grounding claim.

Even though it becomes more apparent in the existential paraphrase because it exposes the commitments of grounding claims more clearly, the original version faces essentially the same problem. It is also committed to there being an additional entity which involves all of the entities the complex concept involves and which the complex concept metaphysically depends on. We have no independent reason to assume that this is the case as the tuple hardly seems relevant to or required for the complex concept. However, the intermediate step, the tuple-detour, is not introduced because it is in itself plausible. It is introduced in order to perform certain 
theoretical tasks. As long as these tasks are properly performed, one might after all be content with the concept grounding view.

\subsection{The Lack of Support for Concept-Grounding}

The main problem with the concept grounding view is that concept-grounding alone fails to perform the task it is supposed to perform. As we have seen, concept-grounding is supposed to be the best way to understand the intuitive hierarchy of concepts, which we investigate in analyses. Concept-grounding then must provide convincing assessments of analysis claims. In some cases the features that come with being a strict partial order suffice to do the job. Let me call such cases "easy cases". Now, an analysis of EQUIANGULAR TRIANGLE reveals EQUIANGULAR rather than EQUILATERAL. The concept grounding view then must hold that EQUIANGULAR TRIANGLE is grounded in EQUIANGULAR and not in EQUILATERAL. Easy as this case might seem, it is not an easy case in the sense just given. It is a hard case because the structural features of grounding do not suffice.

For hard cases, it seems that the correct grounding claims, i. e. the ones that should come out, are always the "plausible" ones while the incorrect ones can be ruled out as "implausible": "intuitively, the concept TRIANGULARITY is not grounded in the concept TRILATERALITY" (Horvath, 2018, 742). One can certainly agree with the particular assessment but one should also wonder why one agrees.

One option is that we have direct intuitive access to the underlying grounding relation. Of course, we cannot simply treat our existing hierarchy-intuitions as grounding-intuitions in order to argue that we should understand the hierarchy in terms of grounding. Without an independent reason to assume that our hierarchyintuitions in fact are grounding-intuitions, we would merely relabel our intuitions about what should come out in an analysis. However, maybe we have additional, novel intuitions for concept-grounding. Whether we do or whether the intuitively clear cases are in fact settled by intuitions about another relation, such as conceptcomposition (see Sect. 4), is hard to assess. But even if we had some direct intuitions for concept-grounding, this would not sufficiently support the concept grounding view. As I have argued, while some of the grounding claims may enjoy intuitive support, many fail to do so. In particular, the tuple-detour requires the concept grounding view to systematically make unintuitive grounding claims. The claims what a given concept is immediately grounded in are not supported by intuitions. If intuitions were our main guide, they would, arguably, be ruled out. So intuitions alone cannot support concept-grounding in the generality required for the concept grounding view to succeed.

What is missing so far is an answer to the questions of how grounding takes place that allows us to better understand why certain concept-grounding claims are fine while others are not. Friends and foes of grounding tend to agree that little is achieved by assuming grounding without also specifying how grounding takes place. Friends, such as Schaffer $(2016)$ and Trogdon $(2018,2020)$ disagree with foes, such as Wilson (2014), on whether the demand to support one's grounding claims can be met, not on whether it is reasonable. 
One important way to explain how grounding takes place is by appeal to general principles. General principles play an important role in discussions of the structure of grounding. Grounding-explanations are often assumed to have a tripartite structure (e. g., in Schaffer, 2017; Trogdon, 2018). Besides the ground and the grounded, there also is a mechanism or link involved such as a metaphyiscal principle that brings us from the ground to the grounded in a similar sense in which causal laws bring us from cause to effect. Suppose we want to justify that the truth that this snapper is red is grounded in the truth that it is vermilion. We can appeal to the plausible general principle that instantiations of determinables are grounded in instantiations of their respective determinates. In this case we do not rely on bare grounding but also on the determinatedeterminable relation for support. Suppose we want to justify that the existence of the Andes is partially grounded in the existence of the Chimborazo. We can appeal to the general grounding principle that the existence of wholes is grounded in the existence of their parts. In this case we do not rely on bare grounding but also on the part-whole relation for support. Often the link involves a further relation between the entities involved in the ground and the grounded such as the part-whole relation or the determinate-determinable relation. In order to understand why some concepts are grounded in others, a promising strategy then is this: we appeal to a general principle that explains in terms of a more familiar relation why some concepts are grounded in others.

The natural candidate for this relation is concept-composition. It is a very attractive relation for this task because we would not require a special link. We can simply rely on the general principles we already assume in order to account for composite objects in terms of their components. If complex concepts stand in mereological relations to their components, then it is the same link that secures the grounding of (the existence of) wholes in (the existence of) their parts. So a very natural way to support the claim that EQUIANGULAR TRIANGLE is grounded in EQUIANGULAR rather than EQUILATERAL is: EQUIANGULAR is a part of EQUIANGULAR TRIANGLE while EQUILATERAL is not. But for proponents of the concept grounding view it is unavailable to rely on concept-composition, since part of the motivation offered in favor of conceptgrounding is that it allows us to account for analysis in a less committing way than concept-composition.

While I focus on concept-composition in particular, this problem is more general. Relying on any further relation with the right features undermines concept-grounding as the best candidate to complete our accounts of analysis. If we need another relation to explain how concept-grounding takes place, then this relation has to be a strict partial order with a sensitivity for relevant fundamentality. But with these features it qualifies for the task concept-grounding is invoked for in the concept grounding view. It qualifies and it is not more committing than concept-grounding because concept-grounding needs this relation as support. Regardless of exactly which relation we rely on in order to understand how concept-grounding takes place, concept-grounding is not the best candidate to account for analysis. As this was the motivation to assume concept-grounding, the concept grounding view provides no convincing reason to believe in concept-grounding. 


\section{The Merits of Concept-Composition}

The failure of the concept grounding view only shows that concept-grounding cannot account for conceptual analysis on its own and for this reason cannot be motivated by applying a certain strategy. If grounding and composition, however, are not treated as rivals, they complement each other naturally. The notion of composition fulfills two important tasks. It directly accounts for the intuitive hierarchy of concepts: what we reveal in an analysis are components and the way they need to be arranged in order to compose complex concepts. It also gives us a reason to believe in concept-grounding insofar as it plausibly serves as the underlying relation. The idea is straightforward if we assume concept-composition to be a mereological relation:

A1 The existence of wholes is grounded in the existence of their parts. ${ }^{8}$

A2 Complex concepts have simpler concepts as their parts.

C Therefore, the existence of complex concepts is grounded in the existence of simpler concepts.

This gives us one important and plausible connection between conceptual analysis and grounding. As conceptual analyses reveal what the parts of concepts are, they also reveal the grounds (for the existence) of complex concepts. However, the relation which directly accounts for the intuitive hierarchy of concepts is composition rather than grounding.

My aim so far was to argue that invoking concept-grounding as alternative to concept-composition does not suffice to account for analysis. Since part of my objection against the concept grounding view is that we can rely on concept-composition instead, I have to at least indicate how we can account for analysis in terms of composition, which includes fending off two objections raised by the concept grounding view. I will do so with a focus on mereological composition. As I focus on plausible roles for grounding in analysis, I cannot develop and defend a theory of non-material objects as structured wholes here and apply it to concepts. ${ }^{9}$ Instead I will appeal, often by means of analogy, to our core understanding of parthood. Let me start with the main objection against a mereological understanding of complex concepts (Horvath, 2018, 736) ${ }^{10}$ which rests on two assumptions such an understanding seems committed to:

\footnotetext{
${ }^{8}$ For a voice dissenting about a particular case, the cosmos, see Schaffer (2010). For a voice dissenting, inter alia, about concepts, see Leibniz (1996, 145), Leibniz et al. (2013, 327). For Leibniz, mereological priority relations are reversed for ideal objects, such as concepts, in contrast to material ones.

${ }^{9}$ For a booklong treatment of material objects as structured wholes see Koslicki (2008). A careful discussion of concepts as mental types with mereological parts is Davis (2003, chs. 12-14). For concepts as abstract senses, the most detailed discussion still can be found in Bolzano (1837, vol. I, §56-65, p. 243296). Of particular interest is the argument for mereologically simple concepts in terms of grounding (\$61, p. 263f, see Schnieder, 2020).

${ }^{10}$ This problem as a problem for mereological conceptions of concepts is already present in Leibniz (Mates, 1986, 61f) and it has been explicitly pointed out by Bolzano (1978, 148f); see Künne (1997, 223-226) and (2001 [2008]) for discussion).
} 
(1) Some complex concepts need to have the same concept as part more than once.

(2) Complex concepts cannot have the same concept as part more than once.

Let us suppose we have a concept $A=_{d f} \neg B \wedge \neg C$. According to (2), arguably an instance of a mereological truism (but see Bennett 2013), I cannot say that A is to be analyzed into a set of concepts in which one and the same negation occurs twice. Nor should I prefer a token-reading of "the same concept" and claim that the concept is to be analyzed into a set that contains two distinct tokens of the same concept-type, as we would have no means to distinguish them. But, what is the motivation for (1), it seems that I have to say one of these things, for otherwise I could not distinguish the analysis of $A$ from the analysis of $F=_{d f} \neg B \wedge C$.

The objection successfully shows that concepts should not be mistaken for the mereological sums of their parts. It then is an objection against understanding complex concepts as wholes in extensional mereology. But structured mereological compounds are generally not to be mistaken for the unstructured sums of their parts (cf. Sanford, 2003; Koslicki 2008), which does not prevent us from understanding such objects in terms of part and whole. If concepts are structured, we acknowledge that the components alone do not suffice to form a complex concept. They also must be combined in the right way. Although sometimes a bit improper, we can use quasispatial expressions in order to describe such a structure. We also can do so in terms of the functional roles the parts stand in to each other, which is how I will usually express myself. That VIXEN can be analyzed into FEMALE FOX then can be understood as the claim that VIXEN is identical to the result of combining FEMALE, FOX and a conjunction concept such that the latter concept conjoins the former two. Assuming such structures allows us to draw distinctions that allow us to reject both assumptions needed for the objection.

In order to reject the first assumption, we can take a cue from Künne (1997, 225f) and Bennett (2013) in order to explain the appearance of multiple occurrences in a different way. We often distinguish between the function something has and the something that has this function. One and the same thing can have several functions within the same complex structure, such as Elizabeth II, who has the function of being queen of a member-state 16 times in the British Commonwealth. Distinguishing between functions and their occupants, we then can reject (1) if we assume that one and the same concept also can have several functions in a given concept. In UNFORTUNATE AND UNCOMMON EVENT, in contrast to FORTUNATE AND UNCOMMON EVENT, the negation concept still occurs only once but it has two roles. In both analyses, the same concepts are listed, namely NOT, FORTUNATE, AND, COMMON, EVENT. However, two different structures are revealed. The structure that is revealed by the analysis of the former concept is more complex than that of the latter as we need to apply NOT twice, we have to "come back" to it at some point. This also seems to be the most natural way to describe the composition of the concept. We can intelligibly ask what the negation concept does in the complex concept without being forced to specify which one. And it is a perfectly natural answer that it does two things: it negates FORTUNATE and it negates COMMON.

Alternatively, we can reject the second assumption by wielding the type-token distinction as Davis does in a related context. Davis appeals to the notion of subtypes 
in order to account for multiple occurrences of word-types in sentence-types: these occurrences "are different subtypes with different relative positions in the whole" (Davis, 2014, 361). Depending on whether the role of tokens is already occupied in one's theory of concepts, one might pick subtypes or tokens in order to account for multiple occurrences of concepts. The idea remains the same: there indeed are multiple occurrences of the negation concept in UNFORTUNATE AND UNCOMMMON EVENT. These occurrences are multiple distinct tokens or sub-types of the same concepttype. And we can distinguish them by appeal to their relation to other parts of the concept: one of these occurrences modifies (or is attached to) COMMON while the other one modifies (or is attached to) FORTUNATE.

These are two generally available ways to understand multiple occurrences. They should not become unavailable just because the complex objects here are concepts. ${ }^{11}$ Which of them one prefers is not to be settled here as each gives us the means to counter the objection.

In order to justify my claim that concept-composition can account for analysis, let me now indicate how it achieves the correct results by considering some of the adequacy conditions Horvath discusses (2018, 728-731, 741-743). The cases fall into three categories. The matter is straightforward for easy cases, cases in which the features of a strict partial order yield the correct results, because proper parthood is a strict partial order. We can easily rule out circular analyses such as an analysis of KNOWLEDGE into the concept KNOWLEDGE by appeal to the irreflexivity of proper parthood. Similarly, in order to account for the asymmetry of analysis, we can appeal to the asymmetry of proper parthood.

Most cases are hard cases. Solving hard cases can always appear unsatisfactory because we ultimately need to rely on intuitions concerning the relation we take to underlie analysis. So I admittedly have to assume that we are familiar enough with the notion of proper parthood to intuitively assess whether it obtains between pairs of concepts. Let me start with the familiar example. We all have to account for the fact that, intuitively, EQUILATERAL rather than EQUIANGULAR appears in an analysis of EQUilateral TRIANGLE. Proponents of the concept grounding view then claim that, intuitively, the latter is grounded in EQUILATERAL rather than EQUIANGULAR whereas I need to appeal to the intuition that it is composed of it. To give two more examples of the claims I need to be plausible enough (Horvath, 2018, 742f): in order to rule out the analysis KNOWLEDGE ${ }_{d f}$ ERNEST Sosa's ACTUAL FAVORITE PHILOSOPHICAL TOPIC, a case of irrelevant necessary coextension, it has to be plausible enough that ERNEST Sosa is not a part of KNOWLEDGe. We also have to distinguish the analyses

\footnotetext{
${ }^{11}$ Both solutions rely on the idea that we can distinguish functional roles or positions of concepts in other concepts, which I will also make use of in what follows. One might worry that this assumption is not independently motivated. However, there certainly are structured complex objects, such as the Commonwealth or an $\mathrm{H}_{2} \mathrm{O}$ molecule (Koslicki, 2008, 5), which we cannot aptly describe by merely listing parts. The parts also need to stand in certain relations to each other, be that their positions to one another or what I called "functions". Is there an independent motivation to assume that concepts are such complexes? As I have already indicated, at least it is natural to describe concepts in this way, such that, e. g, in HINNY, FEMALE combines with DONKEY and MALE combines with HORSE but vice versa for MULE. So I take the assumption to be well motivated as long as we are doing descriptive metaphysics.
} 
of concepts that are trivially necessarily coextensional because they are incoherent, such as ROUND SQUARE and ROUND TRIANGLE. In this case I then have to assume that SQUARE is only part of the former while TRIANGLE is only part of the latter concept, which also enjoys intuitive support.

Finally, we can easily make sense of features that arise from considering the logical complexity of concepts (Horvath, 2018, 740): we want to distinguish the analyses of the concepts $A={ }_{d f}\left(C_{1} \vee C_{2}\right) \vee\left(C_{1} \vee C_{3}\right)$ and $B={ }_{d f}\left(C_{1} \vee C_{2}\right) \vee\left(C_{2} \vee C_{3}\right)$, even though $\mathrm{A}$ and $\mathrm{B}$ are necessarily coextensive and ultimately composed of the same concepts. If we believe in concept-composition, we have a very natural way to account for the difference. Our ordinary notion of parthood allows for more and less fine-grained divisions into parts: we do not have to cut a pizza into eight slices, we can just cut it in half. Despite being composed of the same simple concepts, the analyses of $A$ and $B$ differ in their respective composition because only $A$ has $\left(C_{1} \vee C_{3}\right)$ as one of its bigger parts, which can also be spelled out in terms of different structures.

This feature touches the other objection that is raised against concept-composition: the possibility of multiple equally good analyses can seem problematic for concept-composition (Horvath, 2018, 737). Consider GREEN OR BLUE as plausible analysis of simplified GRUE. If BLUE OR GREEN can equally good serve as an analysis and if these analyses are distinct, then GRUE would have two distinct analyses. These are very substantial "if"s but in case we want to distinguish the disjunctions and want to accept both as analyses of GRUE, concept-grounding is able to handle this because one thing can have multiple full grounds. Does an account based on mereological composition offer us the same theoretical possibility?

Being a structured compound does not generally prevent an object from having several equally good analyses because having distinct ways of combining components does not imply that the combinations are distinct. One way to analyze the Chimborazo is by decomposing it into the summit and the rest while indicating that the former belongs on top of the latter. Another way to decompose it is into its Eastern and its Western half and to indicate their relative positions to each other. In both analyses we are still dealing with the same whole, the Chimborazo, even though we identify distinct parts and ways of combination. At least when analyses also differ in the parts they indicate, as different potential analyses of CIRCLE seem to (Horvath, 2018, 735), we can distinguish different analyses of the same object.

Now if we really also need to assume that BLUE OR GREEN and GREEN OR BLUE serve as distinct analyses of GRUE, then we will probably do so because in one of them the concept BLUE, in some sense, comes first and GREEN later while in the other case GREEN comes first and BLUE later. And this is what we should take the analyses to differ in. While they list the same parts, the indicated structures differ by assumption. I still have to say that GREEN, BLUE, OR combined in one way can be identical to GREEN, BLUE, OR combined in a different way. But this seems unproblematic because ways of combination are, in this scenario, very finely individuated. This still does not mean that the analyses differ in what is analyzed. One way to picture this is that the mediating step of analyzing GRUE in one case would reveal an incomplete disjunction containing GREEN with BLUE missing and vice versa for the other way of analyzing GRUE. 
Given how I have argued against the concept grounding view, I still need to provide an independent motivation for the idea that complex concepts are composite objects. First of all, concept-composition can be motivated as developing a traditional notion, which plays an important role in the history of philosophy. As noted, it has been adopted by Leibniz and Bolzano but it was more influentially spread by Kant's characterization of analyticity (Kant, 1781/1787 [1998], A7/B11). Most importantly, we find it in Locke (1690 [1975], II xxii §9), who was crucial for establishing talk of concepts (or, as Locke prefers, "ideas") in the first place. Even if this usage does not pertain to all traditions, it is an important and established way to think of concepts in the analytic tradition. And this should give us, as long as we are working the field of descriptive metaphysics, some initial reason to understand complex concepts as composite objects. Of course, we can revise this conceptual scheme and we should revise it if another understanding of complex concepts systematized our way of thinking about concepts better. As I have argued, concept-grounding falls short of providing a better understanding.

Secondly, not only do we talk and think in this way, it also is theoretically fruitful to do so. While the notions of composition and containment, as applied to contents such as propositions and concepts, have been attacked in and after Quine (1951), there is a recent interest in them (cf. Correia, 2004; Fine, 2016). As I have suggested in this section, it can provide an understanding of analysis and we can even give proponents of concept-grounding a way to achieve some of their aims. Finally, once the notion of composition is back at the center of our attention, there is a natural way to fruitfully incorporate grounding into our accounts of analysis, to which I will turn now.

\section{Grounding Truths About Concepts}

I have argued that the main work in accounting for analysis is performed by composition rather than grounding. Now let me also propose an important role for grounding in conceptual analysis: grounding explanatorily relates truths about the composition of concepts to truths about their functions.

One important role concepts play is that of being graspables, i. e. of being intersubjective contents of our mental states: we have or possess concepts, we grasp them, employ them in thinking and form thoughts with them (see, e. g., Peacocke, 1992). In this sense, in an analysis we investigate "the structure of thought" (Blackburn, 1999, 2). Focusing on this role, the idea is that by analyzing DRAKE into MALE and DUCK what we reveal of actual interest is that thinking or possessing DRAKE consists in thinking or possessing MALE and DUCK in the right way. Another important role of a particularly important class of concepts is that of being representata (see, e. g., Fodor, 1998). Focusing on this role, the idea is that by analyzing DRAKE into MALE and DUCK what we reveal of actual interest are the application conditions of DRAKE in terms of the application conditions of MALE and DUCK.

The idea is as follows: we begin with the relations that we assume for concepts in order for them to fulfill these tasks. Then we establish grounding connections between the truths that these relations obtain. As a way to understand analysis in 
terms of grounding, this relations first approach has two advantages over the concept grounding view. Firstly, it is less controversial and more natural because it works well with grounding as a relation between truths. It also is cost-efficient as it makes use of the relations we already assume and then establishes an explanatory order between the truths that they obtain. Secondly, it is better suited to make sense out of our practice of analysis. When we analyze a concept, we rarely do so with the aim of revealing what it depends on for its own sake. We want to understand how a given concept performs certain functions in terms of other concepts.

The relations first approach has one apparent drawback: since the relations come first and the grounding comes later, one first has to settle on the relations. Since here is not the place to settle them, this leads to a presentational problem. As I cannot always make perfectly general claims, I will make some very specific assumptions in order to illustrate the approach. In particular, I assume that concepts are mereological compounds and that they are abstract senses in the Fregean tradition. Concerning the relations, I will take some inspiration from Bolzano, who operates in a framework that fits the one I have assumed or argued for so far. He also specifies the functions of concepts with bold claims, which makes them perfectly suited for the purpose of illustration. Given that my aim is only to illustrate an approach, I will not argue for my interpretation or the claims.

\subsection{Grounding Thinking}

Let us first focus on the role of concepts as graspables. In an analysis, we then are interested in how the composition of complex concepts relates to the composition of our acts of thinking them. Consider the following thesis:

Mirroring Hypothesis Grasping a complex concept is a complex mental act. The parts of grasping a complex concept are graspings of the concepts that have the parts of the complex concept that is grasped as their respective contents. (cf. Bolzano, 1837, vol. I, §65, p. 283, vol. III, §281, p. 39)

According to the Mirroring Hypothesis, acts of thinking can be mereologically complex with their structure mirroring the structure of their contents. Of course, the Mirroring Hypothesis is contentious. It and its variants are part of an important debate I cannot possibily do justice to here. Most prominently, they are under attack because the assumed complexity of concepts and acts of processing them fails to show in empirical studies (cf. Fodor et al., 1980; Margolis and Laurence, 1999, 17f). Processing complex concepts, e. g., does not take more time than processing their alleged parts. But defenses are also available: Davis (2003, ch. 14), for example, argues that the duration of acts often fails to be a valid indicator for their complexity. Whatever doubts one might rightly have, the Mirroring Hypothesis should be palatable enough to serve as an example.

Assuming that the structure of certain mental acts mirrors the structure of their contents, this is unlikely to be a happy accident. Instead, the structure of one of them presumably is more fundamental and explains the structure of the other. If we 
conceive of concepts as abstract senses, it is most natural to assume that acts of thinking DONKEY, for instance, obtain in virtue of having the concept DONKEY as their content. The third realm is autonomous but part of what it is to be a grasping of DONKEY is to have DONKEY as its content. The Mirroring Hypothesis together with the assumed priority of the third realm over the second realm then suggests a grounding connection. Accordingly, the truth that the concept VIXEN has as constituents the concepts FEMALE and FOX serves as ground for the truth that grasping VIXEN consists in grasping FEMALE and in grasping FOX in the right way. This suggests the following general grounding principle:

Grounding Thinking If $\mathrm{G}$ is an act of grasping the complex concept $\mathrm{C}$, then $\mathrm{G}$ occurs because $\mathrm{G}$ has parts that are graspings of the parts of $\mathrm{C}$ and these graspings occur. (cf. Bolzano, 1849, 10)

\subsection{Grounding Application Conditions}

If we focus on concepts as representational devices, then the basic idea is similar:

Grounding Application Conditions A complex concept C represents the objects it represents because it is composed in a particular way out of representational concepts $R_{1}, \ldots, R_{n}$ with certain representational features and logical concepts $L_{1}$, $\ldots, L_{n}$ that operate on the representational features of $R_{1}, \ldots, R_{n}$.

DRAKE, for example, represents drakes because it is composed of MALE, DUCK and a conjunction concept; MALE represents all male things, DUCK represents all ducks and the conjunction concept forms the intersection of these two extensions. All application conditions according to the following understanding then are grounded in the composition of a concept:

Application Condition $_{\text {wide }}$ Concept A specifies an application condition of concept $\mathrm{C}$ if and only if something must fall under $\mathrm{A}$ in order to fall under $\mathrm{C}$.

According to this understanding, EQUILATERAL TRIANGLE and EQUIANGULAR TRIANGLE have the same application conditions despite being distinct concepts, among them those specified by EQUILATERAL and by EQUIANGULAR. So concepts that specify application conditions are not exactly what we are after in analyses. One of the merits of the concept grounding view was that it can provide a diagnosis of what goes wrong when we conflate concepts that specify application conditions in the wide sense with what we are after in an analysis. ${ }^{12}$ In a good sense some of the wide application conditions are more relevant than others. The relations first approach allows us to

\footnotetext{
12 See Schnieder $(2010,319)$ for an earlier observation of this common conflation in the 20th century and a brief exposition of the diagnosis as it can already be found in Plato's Eutyphro. For a series of arguments against the conflation which are based on mereological considerations rather than on grounding, see Bolzano (1837, vol. I, §64, 269-282).
} 
clarify this sense in different ways. If we believe that concepts are composite objects and want to know which concepts that specify application conditions are responsible for the whole set of application conditions, then the following specification seems to capture the pertinent notion of narrow application conditions:

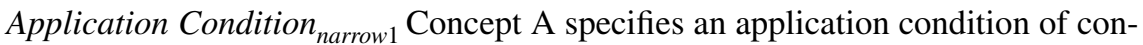
cept $\mathrm{C}$ if and only if something must fall under $\mathrm{A}$ in order to fall under $\mathrm{C}$ and $\mathrm{A}$ composes $C$.

Another understanding, if we focus on the role of concepts as graspables, is the following:

Application Condition $_{\text {narrow } 2}$ Concept A specifies an application condition of concept $\mathrm{C}$ if and only if something must fall under $\mathrm{A}$ in order to fall under $\mathrm{C}$ and one must think $A$ in order to think $C$.

These understandings coincide if the Mirroring Hypothesis is correct. Indeed, one should suspect interrelations because the composition of a complex concept plausibly serves as ground for several of its functions. While a tidy picture such as the one my example principles yield certainly would be attractive, all depends on the specific relations we will settle on, which come first.

\section{Conclusion}

There is a way in which grounding can yield a better understanding of conceptual analysis, however not in the way proposed by the concept grounding view. The negative result is supported by general worries about entity-grounding and the tupledetour but most importantly by the observation that grounding does not simply take place without some kind of support, which the concept grounding view undermines. With a little help from its friends, however, grounding can fruitfully be applied to analysis. One of these friends is the relation of composition, which the concept grounding view treats as rival. I have argued that composition relations between concepts are our natural way of spelling out the intuitive hierarchy of concepts and that the concept grounding view fails to offer a convincing reason to abandon it in favor of concept-grounding. If we rely on concept-composition, we can argue for grounding relations between truths about concepts in order to better understand what we want to understand when we analyze concepts. Among the things we want to understand are further relations: one that holds between concepts and thinkers and one that holds between concepts and their objects. Presumably, there is an explanatory ordering of all these truths and it seems initially plausible that truths about the composition of concepts are more basic than truths about their functions. The most fruitful place for grounding in our accounts of analysis is to sort truths that state the composition of concepts and truths that state their functions with general grounding principles serving as links. It still requires more collaborative work on the relations 
before grounding-metaphysicians can safely take over and figure out the most plausible links.

Acknowledgements For individual feedback at various stages I would like to thank Julio De Rizzo, Yannic Kappes, Stefan Roski and Jonas Werner. Two anonymous referees of this journal helped to greatly improve this paper, for which I am grateful. For financial support I would like to thank the Fritz Thyssen Stiftung for funding the research project "Der Leibniz Böhmens" - die Leibniz'schen Elemente in Bernard Bolzanos philosophischem System.

Funding Open access funding provided by University of Vienna.

Open Access This article is licensed under a Creative Commons Attribution 4.0 International License, which permits use, sharing, adaptation, distribution and reproduction in any medium or format, as long as you give appropriate credit to the original author(s) and the source, provide a link to the Creative Commons licence, and indicate if changes were made. The images or other third party material in this article are included in the article's Creative Commons licence, unless indicated otherwise in a credit line to the material. If material is not included in the article's Creative Commons licence and your intended use is not permitted by statutory regulation or exceeds the permitted use, you will need to obtain permission directly from the copyright holder. To view a copy of this licence, visit http://creativecommons.org/licen ses/by/4.0/.

\section{References}

Aristotle (1984). In J. Barnes (Ed.), The Complete Works of Aristotle (Vols. I \& II.). Princeton University Press.

Audi, P. (2012a). A clarification and defense of the notion of grounding. In F. Correia \& B. Schnieder, (Eds.), Metaphysical grounding (pp. 101-121). Cambridge University Press.

Audi, P. (2012). Grounding: Toward a theory of the in-virtue-of relation. The Journal of Philosophy, 109, $685-711$.

Ball, D. (2020). Revisionary analysis without meaning change. In A. Burgess, H. Cappelen, \& D. Plunkett (Eds.), Conceptual engineering and conceptual ethics (pp. 35-58). Oxford University Press.

Bennett, K. (2013). Having a part twice over. Australasian Journal of Philosophy, 91, 83-103.

Bennett, K. (2017). Making things up. Oxford University Press.

Blackburn, S. (1999). Think: A compelling introduction to philosophy. Oxford University Press.

Bliss, R., \& Trogdon, K. (2016). Metaphysical grounding. In E. N. Zalta, (Ed.), The Stanford encyclopedia of philosophy, winter edition. Metaphysics Research Lab, Stanford University.

Bolzano, B. (1837). Wissenschaftslehre. Seidel, Sulzbach. Edited and translated as Theory of Science (2014) by P. Rusnock and R. George. Oxford University Press.

Bolzano, B. (1849). Was ist Philosophie? Wilhelm Braumüller.

Bolzano, B. (1978). Vermischte philosophische und physikalische Schriften 1832-1848, volume 2A12/2 of Bernard Bolzano Gesamtausgabe. Frommann-Holzboog, Stuttgart. Edited by Jan Berg.

Bryant, A. (2020). Physicalism. In M. Raven (Ed.), The Routledge handbook of metaphysical grounding (pp. 484-500). Routledge.

Cameron, R. (2014). Parts generate the whole, but they are not identical to it. In A. Cotnoir \& D. Baxter (Eds.), Composition as Identity (pp. 90-110). Oxford University Press.

Correia, F. (2004). Semantics for analytic containment. Studia Logica, 77, 87-104.

Correia, F., \& Schnieder, B. (2012). Grounding: an opinionated introduction. In F. Correia \& B. Schnieder (Ed.), Metaphysical grounding (pp. 1-36). Cambridge University Press.

Daly, C. (2012). Scepticism about grounding. In F. Correia \& B. Schnieder (Ed.), Metaphysical grounding (pp. 81-100). Cambridge University Press.

Davis, W. (2003). Meaning, expression and thought. Cambridge University Press.

Davis, W. (2014). On occurrences of types in types. Australasian Journal of Philosophy, 92, 349-363.

Eklund, M. (2015). Intuitions, conceptual engineering, and conceptual fixed points. In C. Daly (Ed.), The Palgrave handbook of philosophical methods (pp. 363-385). Springer. 
Fine, K. (2012) Guide to ground. In In F. Correia \& B. Schnieder (Ed.), Metaphysical grounding (pp. 37-80). Cambridge University Press.

Fine, K. (2015). Unified foundations for essence and ground. Journal of the American Philosophical Association, 1, 296-311.

Fine, K. (2016). Angellic content. Journal of Philosophical Logic, 45, 199-226.

Fodor, J. (1998). Concepts: Where cognitive science went wrong. Oxford University Press.

Fodor, J., Garrett, M. F., Walker, E. C. T., \& Parkes, C. H. (1980). Against definitions. Cognition, 8, 263-367.

Glock, H.-J. (2008). What is analytic philosophy? Cambridge University Press.

Horvath, J. (2018). Philosophical analysis: The concept grounding view. Philosophy and Phenomenological Research, 97, 724-750.

Jenkins, C. (2011). Is metaphysical dependence irreflexive? The Monist, 94, 267-276.

Kant, I. (1781/1787 [1998]). Critique of pure reason. Edited and translated by P. Guyer and A. W. Wood. Cambridge University Press.

Kornblith, H. (2002). Knowledge and its place in nature. Oxford University Press.

Koslicki, K. (2008). The structure of objects. Oxford University Press.

Koslicki, K. (2015). The coarse-grainedness of grounding. Oxford Studies in Metaphysics, 9, 306-344.

Koslicki, K. (2020). Skeptical doubts. In M. Raven (Ed.), The Routledge handbook of metaphysical grounding (pp. 164-179). Routledge.

Künne, W. (1997). Propositions in Bolzano and Frege. Grazer Philosophische Studien, 53, 203-240.

Künne, W. (2001 [2008]). Constituents of concepts: Bolzano vs. Frege. In Versuche über Bolzano (Vol. 21, pp. 211-232). Academia Verlag, Sankt Augustin: emphBeiträge zur Bolzano-Forschung.

Leibniz, G. W. (1989). Philosophical papers and letters (2nd ed.). Kluwer, Dordrecht. Edited and translated by Leroy E. Loemker.

Leibniz, G. W. (1996). New essays on human understanding. Cambridge University Press. Edited and translated by Peter Remnant and Jonathan Bennett.

Leibniz, G. W., Volder, D., \& Burchard, \& Bernoulli, J. (2013). The Leibniz-De Volder Correspondence. Edited and translated by P. Lodge. Yale University Press.

Locke, J. (1690 [1975]). An essay concerning human understanding. Edited by P. H. Nidditch. Oxford University Press.

Loss, R. (2016). Parts ground the whole and are identical to it. Australasian Journal of Philosophy, 94, 489-498.

Margolis, E., \& Laurence, S. (1999). In concepts: Core readings. In E. Margolis \& S. Laurence, (Ed.), Concepts and cognitive science (pp. 3-81). MIT Press.

Mates, B. (1986). The philosophy of Leibniz. Oxford University Press.

Maurin, A., -S. (2019). Grounding and metaphysical explanation: It's complicated. Philosophical Studies, $176,1573-1594$.

Peacocke, C. (1992). A study of concepts. MIT Press.

Pettit, P. (2020). Analyzing concepts and allocating referents. In A. Burgess, H. Cappelen, \& D. Plunkett (Eds.), Conceptual engineering and conceptual ethics (pp. 333-357). Oxford University Press.

Quine, W. V. O. (1951). Two dogmas of empiricism. The Philosophical Review, 60, 20-43.

Raven, M. (2020). Introduction. In M. Raven (Ed.), The Routledge handbook of metaphysical grounding (pp. 1-14). Routledge.

Rosen, G. (2010). Metaphysical dependence: Grounding and reduction. In B. Hale \& A. Hoffmann (Eds.), Modality: Metaphysics, logic, and epistemology (pp. 109-136). Oxford University Press.

Roski, S., \& Schnieder, B. (2019). Fundamental truths and the principle of sufficient reason in Bolzano's theory of grounding. Journal of the History of Philosophy, 57, 675-706.

Sanford, D. H. (2003). Fusion confusion. Analysis, 63, 1-4.

Schaffer, J. (2009). On what grounds what. In D. Chalmers, D. Manley, \& R. Wasserman (Eds.), Metametaphysics (pp. 347-383). Oxford University Press.

Schaffer, J. (2010). Monism: The priority of the whole. The Philosophical Review, 119, 31-76.

Schaffer, J. (2012). Grounding, transitivity, and contrastivity. In F. Correia \& B. Schnieder (Eds.), Metaphysical Grounding (pp. 122-138). Cambridge University Press.

Schaffer, J. (2016). Ground rules: Lessons from wilson. In K. Aizawa \& C. Gillett (Eds.), Scientific composition and metaphysical ground (pp. 143-169). Palgrave Macmillan.

Schaffer, J. (2017). The ground between the gaps. Philosophers' Imprint, 17, 1-26.

Schnieder, B. (2010). A puzzle about 'because. Logique et Analyse, 317-343.

Schnieder, B. (2020). Atomism and fundamentality. Erkenntnis, 85, 551-574. 
Sider, T. (2012). Writing the book of the world. Oxford University Press.

Skow, B. (2016). Reasons why. Oxford University Press.

Thompson, N. (2016). Metaphysical interdependence. In M. Jago (Ed.), Reality making (pp. 38-56). Oxford University Press.

Trogdon, K. (2018). Grounding-mechanical explanation. Philosophical Studies, 175, 1289-1309.

Trogdon, K. (2020). Truthmaking. In M. Raven (Ed.), The Routledge handbook of metaphysical grounding (pp. 396-407). Routledge.

Varzi, A. 2016). Mereology. In E.N. Zalta (ed.) The Stanford encyclopedia of philosophy, winter edition. Metaphysics Research Lab, Stanford University.

Williamson, T. (2007). The philosophy of philosophy. Blackwell.

Wilson, J. (2014). No work for a theory of grounding. Inquiry, 57, 535-579.

Zylstra, J. (2019). The essence of grounding. Synthese, 196, 5137-5152.

Publisher's Note Springer Nature remains neutral with regard to jurisdictional claims in published maps and institutional affiliations. 